\title{
Confirmation of age-related alterations in inhibitory control using a modified minimally delayed oculomotor response (MDOR) task
}

\author{
Paul C Knox ${ }^{\text {Corresp., } 1}$, Dongmei Liang ${ }^{1,2}$ \\ ${ }^{1}$ Department of Eye and Vision Science, Institute of Life Course and Medical Sciences, University of Liverpool, Liverpool, United Kingdom \\ 2 School of Physical Education \& Sports Science, National Demonstration Centre for Experimental Sports Science Education, South China Normal \\ University, Guangzhou, China \\ Corresponding Author: Paul C Knox \\ Email address: pcknox@liv.ac.uk
}

Considerable effort has been made to measure and understand effect of ageing on inhibitory control using a range of behavioural tasks. In the minimally delayed oculomotor response (MDOR) task, participants are presented with a simple visual target step with variable target display duration (TDD), and instructed to saccade to the target not when it appears (a prosaccade response), but when it disappears (i.e. on target offset). Using this task, we recently found higher error rates and longer latencies for correct responses in older compared to younger participants. Here we have used a modified MDOR task, in which participants were presented with static placeholders identifying potential target positions (increasing spatial information), and three TDDs rather than two (reducing temporal predictability). We found that the yield of analysable trials was generally higher with this modified task and in 28 older (mean \pm SD age: $65 \pm 7 y$ ) and 25 younger ( $26 \pm 7 y$ )

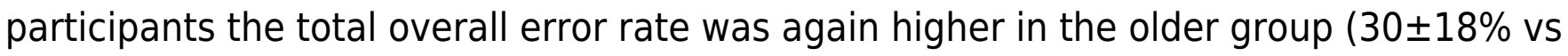
$16 \pm 11 \%)$. An analysis of the temporal distribution of responses demonstrated a pronounced peak in error production around $150 \mathrm{~ms}$ (young) or $200 \mathrm{~ms}$ (old) after target onset. When we recalculated the error rate focusing on these errors, it was again significantly higher in the older group. The latency of correct responses (to offsets) was significantly increased in the older group, although much of this increase was accounted for by expected age-related visuomotor slowing. However, both latency and distribution data suggested that while older participants could generate increased levels of inhibition, they could not maintain these levels as efficiently as the younger participants. In 24 participants (15 old, 9 young) who completed both versions of the MDOR task, neither latency nor error rates differed significantly between versions. These results confirm an inhibitory control deficit in healthy older participants, and suggest that the dynamics of inhibitory control are also affected by ageing. The modified MDOR task yields more data 
while not altering basic performance parameters. 
1 Confirmation of age-related alterations

2

in inhibitory control using a modified minimally delayed oculomotor response (MDOR) task

\author{
Paul C. Knox ${ }^{1}$, Dongmei Liang ${ }^{1,2}$
}

1. Department of Eye and Vision Science, Institute of Life Course and Medical Sciences, University of Liverpool, Liverpool, United Kingdom.

2. School of Physical Education \& Sports Science, National Demonstration Centre for Experimental Sports Science Education, South China Normal University, Guangzhou, China

ORCID ID:

Knox: 0000-0002-2578-7335

Corresponding Author:

Dr Paul C. Knox

pcknox@liv.ac.uk 
26

27

28

29

30

\section{Abstract}

Considerable effort has been made to measure and understand effect of ageing on inhibitory control using a range of behavioural tasks. In the minimally delayed oculomotor response (MDOR) task, participants are presented with a simple visual target step with variable target display duration (TDD), and instructed to saccade to the target not when it appears (a prosaccade response), but when it disappears (i.e. on target offset). Using this task, we recently found higher error rates and longer latencies for correct responses in older compared to younger participants. Here we have used a modified MDOR task, in which participants were presented with static placeholders identifying potential target positions (increasing spatial information), and three TDDs rather than two (reducing temporal predictability). We found that the yield of analysable trials was generally higher with this modified task and in 28 older (mean \pm SD age: $65 \pm 7 y$ ) and 25 younger (26 $\pm 7 y$ ) participants the total overall error rate was again higher in the older group ( $30 \pm 18 \%$ vs $16 \pm 11 \%)$. An analysis of the temporal distribution of responses demonstrated a pronounced peak in error production around 150ms (young) or $200 \mathrm{~ms}$ (old) after target onset. When we recalculated the error rate focusing on these errors, it was again significantly higher in the older group. The latency of correct responses (to offsets) was significantly increased in the older group, although much of this increase was accounted for by expected age-related visuomotor slowing. However, both latency and distribution data suggested that while older participants could generate increased levels of inhibition, they could not maintain these levels as efficiently as the younger participants. In 24 participants (15 old, 9 young) who completed both versions of the MDOR task, neither latency nor error rates differed significantly between versions. These results confirm an inhibitory control deficit in healthy older participants, and suggest that the dynamics of inhibitory control are also affected by ageing. The modified MDOR task yields more data while not altering basic performance parameters. 


\section{Introduction}

54 Stopping ourselves from executing motor actions or thinking thoughts is at least as important as being able to execute those actions or generate those thoughts. Without some means of inhibitory control, both behaviour and cognition become disordered. For this reason, the and measurement, various tasks have been developed to study behavioural inhibitory control 
82 MDOR task by identifying responses to target onsets, which by definition must be inhibition

83

84

85

86

87 failures, an error rate specifically and closely related to the effectiveness of inhibition can be calculated.

We assume that there is a working memory load in the MDOR task; at a minimum there is an instruction to be remembered. But any memory load is reduced compared to both AS and MGS tasks, because it is not necessary to remember a target location; the target is present throughout the period of central fixation prior to target offset. Visuospatial attention is involved in all types of saccade tasks (Kowler et al. 1995) with a close link between the allocation of attention and saccade programming (Deubel 2008). In the AS task attentional resources are divided or distributed between alternative target locations (Klapetek et al. 2016). In most versions of the MGS task, the disappearance of a central fixation target provides the go signal for a saccade directed to the remembered target position, therefore involving two distinct locations and implying some division of attentional resources. In the MDOR task, it is the offset at the target location that provides both the go signal and indicates the position to which the saccade should be directed. Taken together, these considerations suggest that the MDOR task provides a means of investigating behavioural inhibition relatively uncontaminated by those attentional and memory processes that, along with inhibitory control, are key components of executive function (Friedman \& Miyake 2017; Miyake et al. 2000).

We recently used the MDOR task to investigate the effect of normal healthy ageing on behavioural inhibitory control (Knox and Pasunuru 2020). Comparing a group of older participants with previously collected data from younger participants, we found that error rates were higher and the latency of correct responses longer in the older group. Further investigation of the latency differences suggested that most of the increase in latency was accounted for by general age-related slowing in the visuomotor system (Salthouse 1996; Verhaeghen 2011). 
110 However, in the task as used in that study, the stimulus display was sparse, with only simple

111 fixation and saccade targets presented. At the end of each trial, after the eccentric target was

112 extinguished (providing both the go signal and the target location for the saccade), the stimulus

113 monitor was completely blank. This led to a number of potential uncertainties on the part of

114 participants. There was no visual feedback as to the spatial accuracy of their saccade, no

115 marker for how long they should fixate the eccentric position and no formal marker of the end

116 of the trial. This in turn could lead to early blinks and breaks of fixation, and the loss of data. We

117 also only used two TDDs (200ms and $1000 \mathrm{~ms})$ limiting the inferences that could be made with regard to the timing of errors and differences in timing and rates between errors and correct responses. And we were comparing the data collected from older participants with historical data collected in earlier experiments.

We have addressed these and other issues in the current experiment by the addition of static placeholders (at the potential positions of the saccade targets) and the use of three rather than two TDDs. The placeholders reduce the spatial uncertainty in the task; participants have increased information about where to saccade to. The appearance and disappearance of the placeholders also provides a clear indication of the start and end of trials, delineating the intertrial period. The introduction of a third, intermediate TDD (600ms), reduces the temporal predictability of the task, while also proving additional data about the temporal distribution of responses.

In this new experiment, both old and young participants (returning and naïve) have been contemporaneously tested using the modified MDOR task, in order to further investigate task performance and ageing effects reported previously. Retesting previous participants with the new version of the MDOR task (as well as recruiting and testing naïve participants) allowed an investigation of general laboratory or task familiarity effects, as well as an explicit comparison performance stability. 


\section{Materials \& Methods}

\section{Ethics and Participants}

141 Healthy, adult participants, with normal or corrected to normal vision were recruited, under

142 ethical approval from the University of Liverpool Research Ethics committee (Reference

143 number: 2933) and the study was conducted in accord with the ethical standards laid down in

144 the 1964 Declaration of Helsinki. Interested potential participants were provided with a study

145 information sheet. Having read this, and after the experiment was explained to them and they

146 had an opportunity to ask questions, written consent was obtained. Older participants were

147 offered $£ 10$ to compensate them for their time and for the expense of travelling in to the

148 University for testing. Younger participants, who were recruited from the University, were

149 offered $f 5$.

150 Apparatus and Stimuli

151 We used the same apparatus as in previous MDOR experiments (Knox \& Pasunuru 2020; Knox

152 et al. 2018). Briefly, stimuli were presented on a 21 " monitor (1024×768 spatial resolution, 100

$153 \mathrm{~Hz}$ temporal resolution) driven by a VSG2/5 card (Cambridge Research Systems, Rochester, UK),

154 positioned on the fronto-parallel plane $57 \mathrm{~cm}$ from the participant's eye. Horizontal eye

155 position of the left eye was recorded using a Skalar Iris IR Eye Tracker, with the eye tracker

156 output digitised at $1 \mathrm{kHz}$ with 16-bit precision using a CED Power 1401 (Cambridge Electronic

157 Design, Cambridge, UK) interface. Oculomotor data were stored for off-line, trial-by-trial

158 analysis using custom software.

159 Fig 1 illustrates the modified MDOR task used in the present study. As noted in the

160 Introduction, there are two key differences between this version of the task and the version

161 used previously (see Fig $1 \mathrm{~A}$ in Knox and Pasunuru, 2020, for comparison): the inclusion of two

162 target placeholders to the left and right of fixation (within which the saccade target appeared),

163 and the use of three, not two, target display durations (TDD). Each trial began with the

164 appearance of a central fixation target (a $0.2^{\circ}$ black square on a light background) and the two

165 target placeholders. The place holders were themselves $0.8^{\circ}$ square boxes centred $5^{\circ}$ from the

166 fixation target. The fixation target was presented for a randomised period of $0.5 \mathrm{~s}$ to $1.5 \mathrm{~s}$. A 
167 synchronous MDOR task was used in which, when the fixation target was extinguished, the

168

169

170

171

172

173

174

175

176

177

178

179

180

181

182

183

184

185

186

187

188

189

190

191

192

193

194

saccade target appeared at the centre of one of the placeholders ( $5^{\circ}$ from the fixation target) and was displayed for $200 \mathrm{~ms}, 600 \mathrm{~ms}$ or $1000 \mathrm{~ms}$. The offset of the target was the go signal for the saccade.

\section{Procedures}

At the beginning of each testing session, the task was explained to the participant by stepping through it, before a number of trials were run at actual speed. Participants were then carefully positioned by adjusting table height, chin rest and cheek pads, and the eye tracker was applied and adjusted as necessary. Participants were instructed to maintain fixation centrally, until the target in one of the placeholders was extinguished. Then they were to saccade to the centre of the empty placeholder previously occupied by the target, and to maintain fixation there until both placeholders were extinguished (after 1s), leaving the monitor blank. It remained blank until the beginning of the next trial. Participants were asked to refrain from blinking except during the intertrial interval when the monitor was blank. Practice trials were run; these data were not retained. Each participant then completed two runs of 150 MDOR trials (in the original version of the task the run length was 120 trials) with a break in between them. The quality of performance was carefully monitored to ensure that it was maintained, with verbal feedback given as necessary.

In order to calibrate eye tracker output, at the end of each MDOR run, a 32-trial calibration procedure was performed (again the same procedure as used previously; Knox \& Pasunuru 2020; Knox et al. 2018). Calibration trials were simple prosaccade trials in which, after a randomised fixation period (0.5-1.5s), the fixation target was extinguished and a saccade target was presented to the left or right at an eccentricity of $5^{\circ}$ or $10^{\circ}$ (randomised and with equal frequency) for 1s. Participants were instructed to fixate the central point and saccade to the target as soon as it appeared, fixating it until it was extinguished, at which point they could blink and return to the centre, ready for the next trial. All older participants also completed the Addenbrookes Cognitive Examination (ACE) III questionnaire (Hsieh et al. 2013).

\section{Analysis}


195 Oculomotor data were analysed, as previously (Knox \& Pasunuru 2020; Knox et al. 2018), using 196 an interactive program which displayed the eye position data and the time at which the "go" 197 signal (target offset in MDOR trials) occurred. The calibration data were used to transform the 198 data from arbitrary system units into units of degrees of eye rotation. Trials with blinks or unstable fixation prior to target appearance were removed from the analysis. For each valid MDOR trial, the latency (the time from target offset to saccade onset) and amplitude of the primary target-directed saccade were measured.

Data were collated in MS Excel. Any target directed saccade with an amplitude greater than $1^{\circ}$ that occurred from $80 \mathrm{~ms}$ after target onset to $80 \mathrm{~ms}$ after target offset was counted as an error. Error responses were removed and collated separately from correct trials, and the error rate calculated. Any target directed saccade occurring from $80 \mathrm{~ms}$ to $1000 \mathrm{~ms}$ after target offset was counted as a correct response. For each participant, median correct saccade latency was calculated along with the error rate for each TDD. We also calculated the overall total error rate collapsing across TDDs and subsequently derived other error rates, as described in the Results section. Across participant groups, latency and error rate were summarised using the mean. In order to obtain an estimate of prosaccade latency, we calculated median saccade latency from each participant's calibration runs, collapsed across direction, eccentricity and run.

Statistical analysis was conducted with SPSS v25. Repeated measures ANOVA was used to compare groups and conditions with $\eta_{p}{ }^{2}$ reported for effect size (details in Results). Cohen's $d_{s}$ (Lakens 2013) is also reported where relevant.

\section{Results}

\section{Participant characteristics}

A total of 53 participants were recruited and tested in this study. Of 28 older participants (17 male; mean \pm SD age: $65 \pm 7 y$; the "old" group), 16 were naïve to testing and 12 had taken part in our previous study on ageing. The mean ACE III total score for the older group of 28 participants was 95 (range 89-100). The cut-off for suspicion of neurological disease is a score of 88 (Noone 
222 2015) and the mean score for our group was very similar to that reported in (Hsieh et al. 2013)

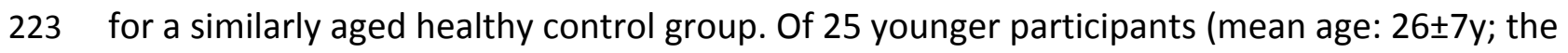

224 "young" group), 21 were naïve to testing and 4 had participated in a previous experiment. A

225 total of 15075 trials were available for analysis (7828 from the old group, 7247 from the young

226 group). The mean trial yield per participant was $93 \pm 8 \%$ and $97 \pm 4 \%$ for the old and young groups

227 respectively $(t=1.98, d f=51, p=0.05)$.

228

229

Analysis of saccade latency

230 The latency of correct MDOR responses across conditions followed the same pattern reported

231 previously (Fig 2). Note that one participant in the old group exhibited extremely high error

232 rates for two of the TDDs leaving fewer than 5 correct trials from which to calculate latency. We

233 therefore removed this participant's data from the latency analysis (leaving $n=27$ ). In both

234 participant groups, raw latency (Fig 2A) was longest in those trials with the shortest TDD

235 (200ms mean $\pm S D$; old: $458 \pm 67 \mathrm{~ms}$, young $370 \pm 67 \mathrm{~ms}$ ) and was clearly modulated by TDD. For

236 both groups there was a monotonic relationship between TDD and latency. For the longest TDD

237 (1000ms) latency was $290 \pm 48 \mathrm{~ms}$ and $257 \pm 34 \mathrm{~ms}$ in the old and young groups respectively. It

238 was consistently longer in the older group by $88 \mathrm{~ms}, 47 \mathrm{~ms}$ and $33 \mathrm{~ms}$ for TDDs of $200,600 \mathrm{~ms}$

239 and $1000 \mathrm{~ms}$ respectively. These latency data were tested with repeated measures ANOVA,

240 treating TDD as within and group as between subjects factors. Both $\operatorname{TDD}\left(F_{2,49}=160, p<0.001\right.$,

$\left.241 \eta_{p}{ }^{2}=0.87\right)$ and group $\left(F_{1,50}=18, p<0.001, \eta_{p}{ }^{2}=0.26\right)$ returned statistically significant results, with a

242 statistically significant interaction between factors $\left(F_{2,49}=6.4, p=0.003, \eta_{p}{ }^{2}=0.21\right)$. The difference

243 between groups for each TDD was statistically significant (t-test with Holm-Sidak correction for

244 multiple comparisons; $p<0.01$ for all pairs) with effect sizes (Cohen's $d_{s}$ ) of 1.17, 0.94 and 0.78

245 for TDDs of $200,600 \mathrm{~ms}$ and $1000 \mathrm{~ms}$ respectively.

246

247 When we compared the latency of reflexive prosaccades in old and young groups (using data

248 from the calibration task), latency in the old group $(209 \pm 44 \mathrm{~ms})$ was significantly longer

249 compared to the young group $\left(181 \pm 41 \mathrm{~ms} ; \mathrm{t}=2.4, \mathrm{df}=50, \mathrm{p}=0.02 ; \mathrm{d}_{\mathrm{s}}=0.66\right)$. Given that this

250 difference represents the general age-related slowing expected for the visuomotor system, we 
251 investigated whether once this was taken into account, MDOR latency still differed between

252 groups. We calculated "corrected" MDOR latency values for each participant by subtracting 253 their median calibration latency from the MDOR latency, and then recalculated MDOR group 254 means (Fig 2B). This had the effect of reducing the latency difference between groups to $60 \mathrm{~ms}$, $25518 \mathrm{~ms}$ and $4 \mathrm{~ms}$ for TDDs of $200 \mathrm{~ms}, 600 \mathrm{~ms}$ and $1000 \mathrm{~ms}$ respectively. When ANOVA was run on 256 these data, the effect of TDD was unchanged $\left(F_{2,49}=160, p<0.001, \eta_{p}{ }^{2}=0.87\right)$, the difference 257 between groups no longer reached statistical significance $\left(F_{1,50}=3.2, p=0.081, \eta_{p}{ }^{2}=0.06\right)$ but 258 there remained a statistically significant interaction between the factors $\left(F_{2,49}=6.4, p=0.003\right.$, $\left.259 \eta_{\mathrm{p}}{ }^{2}=0.21\right)$. Only the difference at $200 \mathrm{~ms}$ was statistically significant $(t=2.96, \mathrm{df}=50$, adjusted 260 $p=0.01$ ) and $D_{s}$ was now $0.83,0.31$ and 0.10 for TDDs of 200,600 ms and $1000 \mathrm{~ms}$ respectively.

In order to quantify and investigate the modulation of latency by TDD, taking all of the data into account, we calculated the least-squares linear regression of latency on TDD for each participant. For both groups this captured the relationship reasonably well, given that the mean $r^{2}$ for these functions was $0.89 \pm 0.12$ and $0.86 \pm 0.12$ for old and young groups respectively. Using the regression slopes to represent the overall modulation in latency, we found that the mean slope for the old group was $-0.21 \pm 0.08$ and was $-0.14 \pm 0.07$ for the young group; this difference between slopes was statistically significant $\left(t=3.5, d f=50, p<0.001, d_{s}=0.99\right)$. To provide comparative data with values reported previously (Knox \& Pasunuru 2020) we also calculated the difference in latency between the 200 ms and 1000ms TDD for each participant; the group means for this difference were $168 \pm 61 \mathrm{~ms}$ and $113 \pm 52 \mathrm{~ms}$ for old and young groups respectively $\left(t=3.5, d f=50, p=0.001, d_{s}=0.97\right)$.

\section{Analysis of errors}

The overall error rate, for all errors calculated across TDD conditions, was considerably higher in the old compared with the young group $\left(30 \pm 18 \%\right.$ vs $\left.16 \pm 11 \% ; t=3.3, d f=44, p=0.002 ; d_{s}=0.93\right)$. When calculated for each TDD, the total error rate $\left(E R_{t}\right)$ was higher in the older group and there was a modulation with TDD (it increased with TDD; Fig 3). When analysed with a similar ANOVA design as used above for latency, TTD returned a statistically significant result $\left(F_{2,50}=94\right.$, 
280

281

282

283

284

285

286

287

288

289

290

291

292

293

$\left.p<0.001, \eta_{p}{ }^{2}=0.79\right)$ as did group $\left(F_{1,51}=10, p=0.002, \eta_{p}{ }^{2}=0.17\right)$, with a statistically significant interaction between the factors $\left(F_{2,50}=7.2, p=0.002, \eta_{p}{ }^{2}=0.22\right)$. The group difference in $E R_{t}$ for each TDD was statistically significant (adjusted $p$ values $200 \mathrm{~ms}: p=0.03,600 \mathrm{~ms}$ and 1000ms:p<0.01), and $d_{s}$ for each TDD was $200 \mathrm{~ms}: 0.67,600 \mathrm{~ms}: 0.99$ and 1000ms:0.81.

The $\mathrm{ER}_{\mathrm{t}}$ captured all the errors as defined in the Methods. We investigated the pattern of errors further by constructing average distributions of all responses for the two participant groups (Fig 4). In each of the six distributions in Fig 4 there are two clear peaks, identified by the arrows. The larger of these is composed of the correct responses, which occur after the target offset (the vertical dashed line at $0 \mathrm{~ms}$ in each plot). The bins prior to $+80 \mathrm{~ms}$ contain error responses, and errors (all of which contribute to the $E R_{t}$ ) occur throughout the period between target onset (at $-200 \mathrm{~ms},-600 \mathrm{~ms}$ and $-1000 \mathrm{~ms}$ ) and target offset. Errors are not distributed uniformly throughout this period, and the second clear feature in these distributions is the early peak just after target onset. The timing of this peak is consistent with these responses being primarily uninhibited responses to the target onset.

Using the average distributions, we defined two further error rates. To capture the early peak, we calculated the proportion of saccades contained in the five histogram bins centred on the peak bin as indicated in Fig 4 (ie covering a latency range of $250 \mathrm{~ms}$ ). This was calculated for each participant individually, and group means then calculated. We will refer to this as the peak error rate $\left(E R_{p k}\right)$. As can be seen from Fig $5 A$ (and consistent with Fig 4), the $E R_{p k}$ was consistently higher in the older group although no longer as clearly modulated by TDD. When tested with ANOVA, the TDD did have a significant effect on the rate $\left(F_{2,50}=43, p<0.001\right.$, $\left.\eta_{p}{ }^{2}=0.63\right)$ as did group $\left(F_{1,51}=6.1, p<0.017, \eta_{p}{ }^{2}=0.12\right)$; the TDD $x$ Group interaction was not statistically significant $\left(F_{1,51}=0.2, p=0.8\right)$. For the $600 \mathrm{~ms}$ and $1000 \mathrm{~ms}$ TDDs, we also defined a "residual" error rate $\left(E R_{r}\right)$. As can been seen from Fig 4, errors continued to occur at a low rate after the peak. While for the shortest TDD $(200 \mathrm{~ms})$ it was difficult to distinguish between the end of the initial peak and the beginning of correct responses to the target offset, this was not the case for the longer TDDs. We therefore used the five bins (the same number of bins used 
309 for the $E R_{p k}$ rate) from $-250 m s$ to $0 m s$ to calculate $E R_{r}$ for the two longer TDDs (Fig 5B), and

310 calculated the proportion of errors contained in these bins for each participant. The difference

311 between groups was now less clear, partly because the average number of errors was relatively

312 low, particularly in the young participants. Statistically, there was now no significant effect of

$313 \operatorname{TDD}\left(F_{1,51}=2.3, p=0.133\right)$, although both the interaction $\left(F_{1,51}=7.2, p=0.01, \eta_{p}{ }^{2}=0.12\right)$ and the

314 group effect were significant $\left(F_{1,51}=8.7, p=0.005, \eta_{p}{ }^{2}=0.14\right)$.

315

316 As indicated by the arrows on Fig 4, the early peaks occurred slightly later in time in the old

317 compared to the young group. To examine the timing of these early error saccades further, we

318 used the bin ranges discussed above (five bins centred on the peak bin in each distribution, a

319 range of $250 \mathrm{~ms}$ ), calculated the median latency of saccades falling in these ranges for each

320 participant and then calculated and compared group means (Fig 6). Saccade latency was longer

321 in the old group as would be expected given the general age-related slowing in the old group

322 noted above. The latency difference was $43 \mathrm{~ms}, 29 \mathrm{~ms}$ and $30 \mathrm{~ms}$ for the TDDs of $200 \mathrm{~ms}, 600 \mathrm{~ms}$

323 and $1000 \mathrm{~ms}$ respectively. When tested with a repeated measures ANOVA similar to those

324 described previously, the difference between groups was statistically significant (Group:

$325 F_{1,47}=51, p<0.001, \eta_{p}{ }^{2}=0.52 ;$ TDD: $F_{2,46}=94, p<0.001, \eta_{p}{ }^{2}=0.80$; interaction: $\left.F_{2,46}=0.4, p=0.68\right)$.

326 Note that there were two young and one old participant in whom no median latency was

327 available for at least one TDD; data from these participants were not included in the ANOVA.

We also investigated whether there was any relationship between each participants' prosaccade latency and the latency of the error saccades comprising the early peak (Fig 7). While the general context in which these prosaccades and MDOR peak error saccades were executed was very different, given that in both cases these were reflexive responses to target onsets, it seemed plausible that there might be a relationship. Overall correlations (in which old and young groups were combined) were calculated (note that in Fig 7 the two groups can be distinguished) for each TDD separately. Correlations were generally low, although for the 200ms and 1000ms TDDs they reached statistical significance. As can be seen from both Figs 6 and 7, latency is lowest for the longest TDD, following the same general pattern observed for 
338

339 340

341

342

343

344

345

346

347

348

349

350

351

352

353

354

355

the correct responses. However, the peak error latency tended to be lower than the prosaccade latency from the calibration task; this is particularly marked for the $1000 \mathrm{~ms}$ TDD (Fig 7C).

In order to assess whether the two participant groups might adopt fundamentally different strategies, sacrificing speed of response for more effective inhibition, we examined the data for evidence of speed/accuracy trade-offs (Fig 8). Statistically there were no significant correlations between either $E R_{t}$ and latency in either group (young Fig $8 \mathrm{~A}$; old Fig $8 \mathrm{C}$ ) or $\mathrm{ER}_{\mathrm{pk}}$ and latency (young Fig 8B; old Fig 8D). Thus there was no evidence of a difference in strategy in the two groups. However, there did appear to be a systematic difference in both groups between the shortest and longest TDDs. For the shortest TDD (200ms) error rate varied widely over a relatively restricted latency range, whereas for the longest TDD $(1000 \mathrm{~ms})$ latency appeared to be more variable.

\section{Comparison of original and modified MDOR tasks}

We sub-divided the old group into those participants who were naïve to testing $(N ; n=16)$ and those who participated in a previous experiment and returned for testing with the modified MDOR task ( $R ; n=12)$. These two groups were comparable in terms of age ( $N: 65 \pm 7 y ; R: 64 \pm 6 y)$

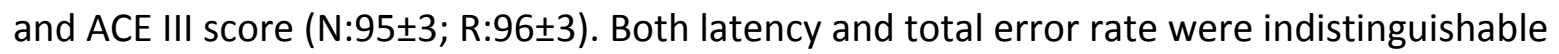
between these groups (Fig 9).

There were a total of 24 participants (15 old, 9 young) for whom data were available both from the original MDOR task (MDOR-O) and the new modified task (MDOR-M). Of the 15 old participants, 12 completed MDOR-O first and MDOR-M second; only 3 completing the tasks in the opposite order. In the young group 4 did MDOR-O first. One of the key differences between the two versions of the task was that in MDOR-O there were only two TDDs (200ms and $1000 \mathrm{~ms})$, so these were the conditions for which comparisons could be made. As is clear from Fig 10, both latency and total error rate were very similar in the two tasks whether considering data from all the participants in an omnibus analysis (Latency - Fig 10A; Total Error Rate - Fig 10D), or separately analysing data from young (Latency - Fig 10B; Total error rate - Fig 10F) and 
367 old (Latency - Fig 10C; Total error rate - Fig 10E) groups. We subjected the latency and error 368 rate data to separate ANOVA's of the same design, which treated TDD as a within and task (M vs O) as between subjects factors. Unsurprisingly the task factor returned a statistically nonsignificant result for both latency $\left(F_{1,46}=2.0, p=0.13\right)$ and error rate $\left(F_{1,46}=0.12, p=0.74\right)$.

\section{Discussion}

Our objectives in the current experiment were to investigate the use of a modified MDOR task and further examine the effects of normal ageing that we had observed with the original task. The MDOR task was originally conceived to provide a means of investigating behavioural inhibitory control using eye movements (Wolohan \& Knox 2014). At that time, and subsequently (Knox \& Pasunuru 2020; Knox et al. 2018); see also the Introduction) we discussed the advantages of the MDOR task compared to a number of alternatives. Essentially this is that it provides a less contaminated measure of the ability of participants to inhibit a prepotent response compared to those alternatives. Potentially this means that it might be a useful tool for detecting and investigating the breakdown in inhibitory control reported to occur in a range of conditions and pathologies (Kaiser et al. 2018; Manza et al. 2017; Rabi et al. 2020; Rochat et al. 2013; Smith et al. 2014).

In the modified MDOR task, we increased spatial information available to participants and decreased temporal predictability compared to the original version, and provided better definition of trial start, end and the intertrial interval. One advantage flowing from these changes appears to be an improved yield of valid trials for analysis. In our previous experiment the mean yield per participant was $78 \%$ and $83 \%$ for young and old participants respectively (Knox \& Pasunuru 2020); here the comparable proportions were $97 \%$ and 93\%. Participants reported that the modified task provided them with more confidence as to how long to fixate the target position towards the end of each trial; in the original version they were simply told to pause at the eccentric position. They also had more confidence about when they could blink because the intertrial interval was now clearly delineated. Much of our analysis focusses on error trials as these provide information specifically about inhibition failure. However, errors 
396

397

398

399

400

401

402

403

404

405

406

407

408

409

410

411

412

413

414

415

416

417

418

419

420

421

422

423

424

make up a minority of trials, and absolute numbers per participant can be small (particularly in younger participants). So task design features that increase the information available for analysis, without fundamentally altering task properties, are valuable.

The general pattern of responses observed with the modified MDOR task was identical to that reported previously (Knox \& Pasunuru 2020; Knox et al. 2018; Wolohan \& Knox 2014). Latency was related to TDD (longer latency at shorter TDDs), as was $\mathrm{ER}_{\mathrm{t}}$ (lower at shorter TDDs), and latency was longer and error rates higher in older compared to younger participants. As previously, most of the latency increase in the older group was due to general age-related slowing in the oculomotor system, replicating our previous result (Knox \& Pasunuru 2020). Prosaccade latency was obtained from the task used to calibrate eye tracker output rather than from a separate prosaccade task, in part to reduce the testing burden on participants. The calibration task is clearly different to the MDOR task, but it provided us with an estimate of prosaccade latency in a consistent manner for all participants. While a different prosaccade task might have produced different absolute latencies, we would expect a similar latency difference between old and young groups. The difference we did observe $(28 \mathrm{~ms})$ was broadly consistent with (although smaller than) the difference we reported previously $(47 \mathrm{~ms})$, and consistent with other reports in the literature (Eenshuistra et al. 2004; Klein et al. 2000). It provided us with a means of "correcting" for general age-related slowing, a procedure recommended in the literature (Verhaeghen 2011, although see also Ulrich et al. 1999). This is important because general slowing accounts for some of the age-related alterations in performance observed across tasks (Salthouse 1996), particularly those associated with executive function (Maldonado et al. 2020). Notably after the latency "correction" a larger TDD-related modulation of latency still remained in the older group, but now measured over three TDDs rather than two (compare Fig 3b in Knox \& Pasunuru 2020 with Fig 2B).

When the response to a target onset is successfully inhibited (so that there is no error response), the result is a correct MDOR response. We have argued that the latency of these correct MDOR responses reflects the level of inhibition around the time the target for the 
425 saccade is extinguished (the go signal). At a TDD of $200 \mathrm{~ms}$, levels of inhibition at target offset

426 are high, leading to long latencies; as TDD is increased the level of inhibition (at the time the

427 correct saccade has to be initiated) falls, and so does latency. Our data suggest that older

428 participants are capable of exerting high levels of inhibition initially. As can be seen from Fig 2B,

429 at TDD $=200 \mathrm{~ms}$ the older group exhibited an additional latency increase, even once general age-

430 related slowing was accounted for; the effect size $\left(d_{s}\right)$ for the difference between groups at

$431200 \mathrm{~ms}$ in the corrected data was 0.83 and was statistically significant. At TDD=600ms this

432 additional latency was greatly decreased (and was not statistically significant), and it was

433 entirely absent at $1000 \mathrm{~ms}$.

434

435 The larger modulation in latency by TDD in the older group, a result we reported previously

436 (Knox \& Pasunuru 2020), suggests that the maintenance of inhibition is more difficult for older

437 participants. The magnitude of the modulation is very similar in both studies; previously

438 comparing $200 \mathrm{~ms}$ and $1000 \mathrm{~ms}$ TDDs the latency difference was $164 \mathrm{~ms}$ and $96 \mathrm{~ms}$ in old and

439 young groups, here is was $168 \mathrm{~ms}$ and $113 \mathrm{~ms}$. We might speculate that initially older

440 participants are able to generate high levels of inhibition, perhaps even higher levels than

441 young participants, as a strategy to compensate for poorer overall effectiveness of inhibition

442 (reflected in generally higher error rates); this would produce the longer latency for correct

443 responses at 200ms TDD. However, an inability to maintain inhibition as TTD increased, would

444 lead to latency dropping off, and a larger overall modulation.

445

446

Independent support for the general hypothesis that the MDOR task evokes inhibition, and that

447

this is reflected in latency increases, is provided by the results of Tari et al. (2019) and Tari \&

448

Heath (2019). They used the MDOR task in a task switching paradigm and found that

449

prosaccades following an MDOR task had increased latency. As with task switching costs

450

observed with mixed AS and prosaccade tasks, they interpreted this as being due to the effects

451

MDOR-induced inhibition.

452 
453 While the latency of correct responses may reflect levels of inhibition, error rates reveal its 454 effectiveness; these were consistently higher in the old compared to the young group.

455 However, the distributions (Fig 4) indicate that it is important to distinguish between different 456 types of error. We have previously discussed the advantages of average, compared to pooled, 457 distributions for prosaccade (Knox \& Wolohan 2014; Knox et al. 2017), antisaccades (Knox et al. 458 2012) and MDOR tasks (Knox \& Pasunuru 2020; Wolohan \& Knox 2014). Here we have used 459 them as a means of graphically summarising responses across groups, in a form which allows 460 the identification of significant features. For errors, the clearest feature is the peak occurring 461 just after target onset, which we used to derive the peak error rate $\left(E R_{p k}\right)$. We suggest that this

462 provides a direct index of inhibitory failure as given their timing, the errors which make up this peak are most likely uninhibited responses to target onsets. Note that a similar claim might be made for antisaccade errors (i.e. that AS errors are uninhibited prosaccades). However, as outlined in the Introduction, this interpretation is complicated by there being two processes competing for behavioural expression in the AS task. An AS error therefore is not necessary the result of a failure of inhibition. In the MDOR task there is no such competition.

However, if early MDOR errors were only uninhibited responses to the target onset, then there is no reason to expect $E R_{p k}$ to differ with TDD. It is striking that in both participant groups $E R_{p k}$ is noticeably raised in the 1000 ms condition (Fig 4C,F; see also Fig 5A) compared to the two other TDDs where indeed it is similar. It seems unlikely that this is a chance occurrence, particularly as it was observed in the distributions in our previous study in both young and old groups (Knox \& Pasunuru 2020), Fig 5B,D). This might suggest some other process influencing $E_{\mathrm{pk}}$ at least when TDD is long. However, supporting the idea that these errors are responses to target onsets (and are therefore for the most part inhibitory failures), the latency difference between groups was similar to that observed between the prosaccade and MDOR correct response latencies (Fig 6), and there was a (modest) correlation between the peak error and prosaccade latency across participants (Fig 7). 
481 The distributions also provide further information about the dynamics of inhibition and its 482 difference between groups. Errors continued (at a low rate) throughout the period when 483 central fixation was required, up to the target offset. But for the 1000ms TDD (Fig 4C,F), the 484 error rate rose slowly as the fixation period wore on, particularly from around $-400 \mathrm{~ms}(600 \mathrm{~ms}$ 485 after the target onset). With TDD $=600 \mathrm{~ms}$ (Fig 4B,E) the error rate was flat in the young group 486 and falling in the old group. The residual error rate (ER $;$ Fig 5B), differed for 600ms TDD but not 487 1000ms TDD, consistent with the young participants being able to maintain inhibition at a 488 higher level for longer than the older group. The absolute number of errors at these timings is 489 small, so these patterns should be interpreted cautiously. But perhaps they provide evidence 490 that the increased inhibition initially evoked in the MDOR task at target onset can only be 491 maintained for a few hundred milliseconds, and that the ability to maintain inhibition declines 492 as we age.

493

If essentially the same processes (or their failure) accounted for both the error rates and the latency of correct responses, then we would expect to see a relationship between them. A participant who was able to exert high levels of inhibitory control might have a low error rate (particularly a low $\mathrm{ER}_{\mathrm{pk}}$ ) and a longer latency for correct responses. There was no clear evidence of this type of relationship across conditions and groups (Fig 8). What the data in Fig 8 do suggest is a difference between the shortest and longest TDDs, but with a similar pattern in both groups. At TDD=1000ms for both $E R_{t}$ and $E R_{p k}$, the error rate was highly variable between participants while latency appeared to be less variable. The opposite pattern is seen for TDD $=200 \mathrm{~ms}$. Latency is longer and error rate higher, but the relationship between them is altered; latency appears to be more variable and error rate less variable. As the same general pattern was seen in both old and young groups, these data provide no evidence of a different control strategy between groups.

Among the older participants, we had comparable groups of naïve and returning participants. The experience of having visited the lab and completed the original MDOR task did not 
510 between tests on the two different versions of the task, there was little evidence of learning or 511 exposure effects. Procedures in the lab were of course designed to produce exactly this result

512 by carefully explaining and demonstrating tasks and providing practice trials before data

513 collection began with the aim of achieving stable performance in any one session of testing.

514 Such stability in performance over time does appear to be a general feature of at least simple

515 saccade tasks. In the absence of training (exposure to large numbers of trials over a relatively

516 short period of time), this has been taken to support the concept of an oculomotor phenotype

517 (Bargary et al. 2017; Knox \& Wolohan 2015). Our study was not designed to investigate the

518 stability of MDOR performance over time; longitudinal testing will be needed to demonstrate

519 that MDOR performance provides a means of measuring trait inhibitory control as opposed to

520 providing a means of measuring the inhibitory state at the time of testing.

521

522 As demonstrated in Fig 10 there was little evidence of difference in latency and total error rate

523 either overall (Fig 10A,D) or in either the young (Fig 10B,E) or old (Fig 10C,F) groups when we

524 compared the original and modified MDOR tasks in the same participants. This suggests a

525 degree of stability across versions of the MDOR task and perhaps also a lack of sensitivity of the

526 measured parameters to task context. One concern with the original MDOR task might have

527 been that with only two TDDs (200ms and 1000ms), participants might have been able to

528 predict the time of target offset, generally supressing latency. The addition a the third TDD

529 would be expected to make this less likely. If such an effect were present, then with the

530 modified task a general increase in latency might be expected. However, there is no consistent

531 evidence that this is the case. Using task design to vary performance is, of course, occasionally

532 useful depending on the type of participants being tested. In the AS task, the use of gap AS

533 tasks tends to increase error rates, usefully preventing floor effects in healthy control groups in

534 clinical studies. We have shown previously that gap and overlap effects do not influence MDOR

535 performance (Knox et al. 2018). So too the modifications made in the current study had little

536 effect on performance.

537 
538 As is well recognised, inhibitory control is not a monolithic construct (Aron 2011), several

539 taxonomies have been used to describe it (Rey-Mermet et al. 2018) and many tasks have been

540 used to investigate it. Correlations between tasks tend to be poor (Friedman \& Miyake 2004;

541 Rey-Mermet et al. 2018) and even tasks that might be thought of as being relatively similar (eg

542 manual go/no go and SSRT; AS and MDOR task) may actually involve different mechanisms or

543 processes (Raud et al. 2020; Wolohan \& Knox 2014). The existence and nature of an age-related

544 inhibitory control deficit has been a matter of recent debate (Rey-Mermet \& Gade 2017;

545 Verhaeghen 2011). However, the MDOR task does appear to offer insights into the

546 effectiveness of inhibition and perhaps also its dynamics, at least with respect to oculomotor

547 control. Using the modified MDOR task in the current study, we have confirmed that the

548 effectiveness of inhibitory control declines in healthy ageing. We suggest that the MDOR task

549 (in either original or modified versions) provides a useful means of investigating multiple

550 aspects of inhibitory control.

551

552

Acknowledgements: We are grateful to all the participants who took part in these experiments.

553

554

555

556

557

558

559

560

561

562

563

564

565

566

567

568

569

570

571

572

\section{References}

Alichniewicz KK, Brunner F, Klünemann HH, and Greenlee MW. 2013. Neural correlates of saccadic inhibition in healthy elderly and patients with amnestic mild cognitive impairment. Frontiers in Psychology 4. 10.3389/fpsyg.2013.00467

Aron AR. 2011. From reactive to proactive and selective control: Developing a richer model for stopping inappropriate responses. Biological Psychiatry 69:e55-e68. http://dx.doi.org/10.1016/j.biopsych.2010.07.024

Bargary G, Bosten JM, Goodbourn PT, Lawrance-Owen AJ, Hogg RE, and Mollon JD. 2017. Individual differences in human eye movements: An oculomotor signature? Vision Res 141:157-169. 10.1016/j.visres.2017.03.001

Bari A, and Robbins TW. 2013. Inhibition and impulsivity: Behavioral and neural basis of response control. Progress in Neurobiology 108:44-79. http://dx.doi.org/10.1016/j.pneurobio.2013.06.005

Castiglione A, Wagner J, Anderson M, and Aron AR. 2019. Preventing a thought from coming to mind elicits increased right frontal beta just as stopping action does. Cerebral Cortex 29:2160-2172. 10.1093/cercor/bhz017

Crawford TJ, Higham S, Renvoize T, Patel J, Dale M, Suriya A, and Tetley S. 2005. Inhibitory control of saccadic eye movements and cognitive impairment in Alzheimer's disease. Biological Psychiatry 57:1052-1060. http://dx.doi.org/10.1016/j.biopsych.2005.01.017 
573 Deubel H. 2008. The time course of presaccadic attention shifts. Psychological Research 72:630-640. 
621

622

623

624

625

626

627

628

629

630

631

632

633

634

635

636

637

638

639

640

641

642

643

644

645

646

647

648

649

650

651

652

653

654

655

656

657

658

659

660

661

662

663

664

665

666

667

668

Maldonado T, Orr JM, Goen JRM, and Bernard JA. 2020. Age differences in the subcomponents of executive functioning. J Gerontol B Psychol Sci Soc Sci 75:e31-e55. 10.1093/geronb/gbaa005

Manza P, Amandola M, Tatineni V, Li C-sR, and Leung H-C. 2017. Response inhibition in Parkinson's disease: a meta-analysis of dopaminergic medication and disease duration effects. $n p j$ Parkinson's Disease 3:23. 10.1038/s41531-017-0024-2

Miyake A, Friedman NP, Emerson MJ, Witzki AH, Howerter A, and Wager TD. 2000. The unity and diversity of executive functions and their contributions to complex "frontal lobe" tasks: A latent variable analysis. Cognitive Psychology 41:49-100. http://dx.doi.org/10.1006/cogp.1999.0734

Munoz DP, and Everling S. 2004. Look away: the anti-saccade task and the voluntary control of eye movement. Nat Rev Neurosci 5:218-228.

Noone P. 2015. Addenbrooke's Cognitive Examination-III. Occup Med (Lond) 65:418-420. 10.1093/occmed/kqv041

Rabi R, Vasquez BP, Alain C, Hasher L, Belleville S, and Anderson ND. 2020. Inhibitory control deficits in individuals with amnestic mild cognitive impairment: a meta-analysis. Neuropsychology Review 30:97-125. 10.1007/s11065-020-09428-6

Raud L, Westerhausen R, Dooley N, and Huster RJ. 2020. Differences in unity: The go/no-go and stop signal tasks rely on different mechanisms. Neurolmage 210:116582. 10.1016/j.neuroimage.2020.116582

Reuter B, Jäger M, Bottlender R, and Kathmann N. 2007. Impaired action control in schizophrenia: The role of volitional saccade initiation. Neuropsychologia 45:1840-1848. http://dx.doi.org/10.1016/j.neuropsychologia.2006.12.006

Reuter B, Rakusan L, and Kathmanna N. 2005. Poor antisaccade performance in schizophrenia: An inhibition deficit? Psychiatry Research 135:1-10. http://dx.doi.org/10.1016/j.psychres.2004.12.006

Rey-Mermet A, and Gade M. 2017. Inhibition in aging: What is preserved? What declines? A metaanalysis. Psychonomic Bulletin \& Review. 10.3758/s13423-017-1384-7

Rey-Mermet A, Gade M, and Oberauer K. 2018. Should we stop thinking about inhibition? Searching for individual and age differences in inhibition ability. Journal of Experimental Psychology: Learning, Memory, and Cognition 44:501-526. 10.1037/xIm0000450

Rochat L, Billieux J, Juillerat Van der Linden A-C, Annoni J-M, Zekry D, Gold G, and Van der Linden M. 2013. A multidimensional approach to impulsivity changes in mild Alzheimer's disease and control participants: Cognitive correlates. Cortex 49:90-100. http://dx.doi.org/10.1016/j.cortex.2011.08.004

Salthouse TA. 1996. The processing-speed theory of adult age differences in cognition. Psychol Rev 103:403-428. 10.1037/0033-295x.103.3.403

Smith JL, Mattick RP, Jamadar SD, and Iredale JM. 2014. Deficits in behavioural inhibition in substance abuse and addiction: A meta-analysis. Drug and Alcohol Dependence 145:1-33. http://dx.doi.org/10.1016/j.drugalcdep.2014.08.009

Tari B, Fadel MA, and Heath M. 2019. Response suppression produces a switch-cost for spatially compatible saccades. Experimental Brain Research. 10.1007/s00221-019-05497-z

Tari B, and Heath M. 2019. Pro- and antisaccade task-switching: response suppression - and not vector inversion-contributes to a task-set inertia. Experimental Brain Research. 10.1007/s00221-01905686-w

Ulrich R, Mattes S, and Miller J. 1999. Donders's assumption of pure insertion: an evaluation on the basis of response dynamics. Acta Psychologica 102:43-76. https://doi.org/10.1016/S00016918(99)00019-0

Verhaeghen P. 2011. Aging and executive control: Reports of a demise greatly exaggerated. Current Directions in Psychological Science 20:174-180. 10.1177/0963721411408772

Peer] reviewing PDF | (2021:02:58204:1:1:NEW 30 Apr 2021) 
669 Wolohan FDA, and Knox PC. 2014. Oculomotor inhibitory control in express saccade makers.

670 Experimental Brain Research 232:3949-3963. 10.1007/s00221-014-4076-9

671 


\section{Figure 1}

Schematic description of the modified MDOR task used in this study.

(A) Each trial began with the appearance of the central fixation target, and two $0.8^{\circ} \times 0.8^{\circ}$ place holders, centred $5^{\circ}$ to the left and right. After a random period of $0.5-1.5 \mathrm{~s}$ the fixation target was extinguished and the target for the saccade appeared randomly at the centre of either the left or right placeholder, and was displayed for $200 \mathrm{~ms}, 600 \mathrm{~ms}$ or $1000 \mathrm{~ms}$ (randomised). The extinction of the target was the signal for the participant to saccade to the centre of the placeholder in which the target had appeared, and maintain fixation there. After 1s the placeholders were extinguished, at which point the participant was instructed to return fixation to the centre of the display and await the beginning of the next trial. (B) The relative timing of the various events in each trial. Note that the eye trace illustrating a correct response assumes a target display duration of $1000 \mathrm{~ms}$. 

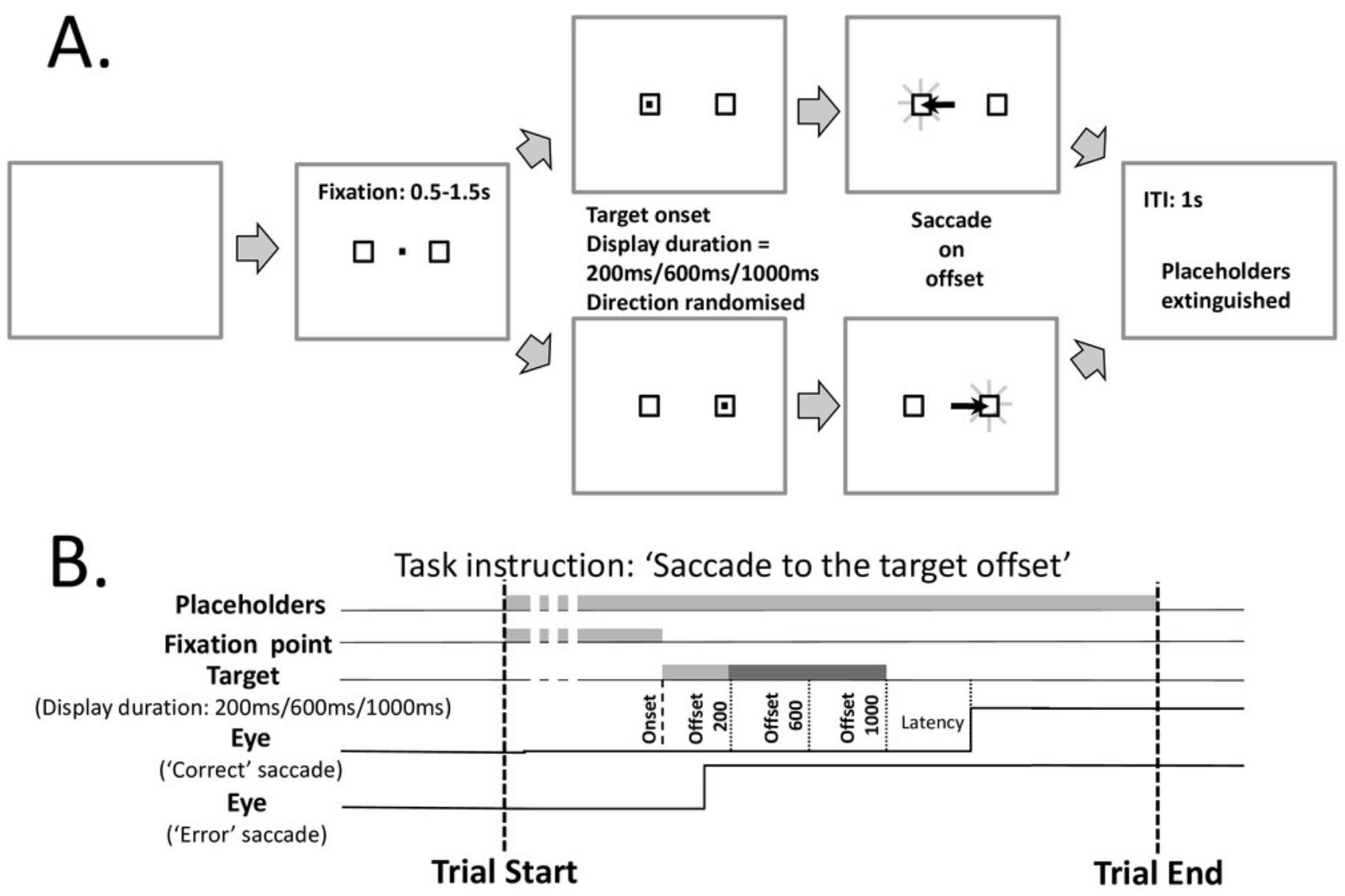
Figure 2

Latency of correct MDOR responses.

Mean $( \pm 95 \% \mathrm{Cl})$ latency is plotted for each target display duration for old and young groups. (A) Uncorrected latency. (B) Corrected latency; each participants calibration task latency was subtracted from their MDOR latency, and group mean latency calculated.

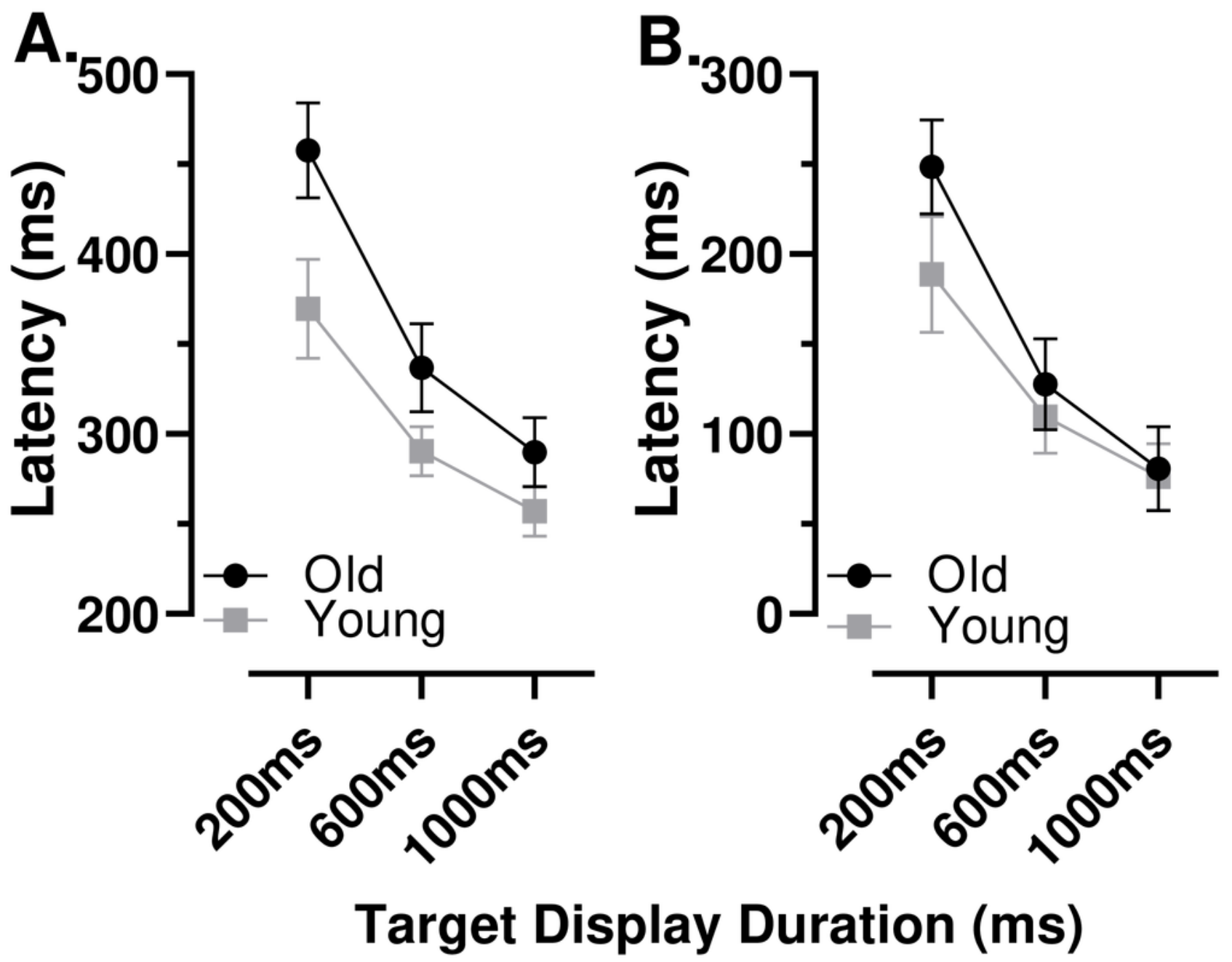


Figure 3

Total error rate.

Mean $( \pm 95 \% \mathrm{Cl})$ total error rate $(\%)$ is plotted for each target display duration for old and young groups. 

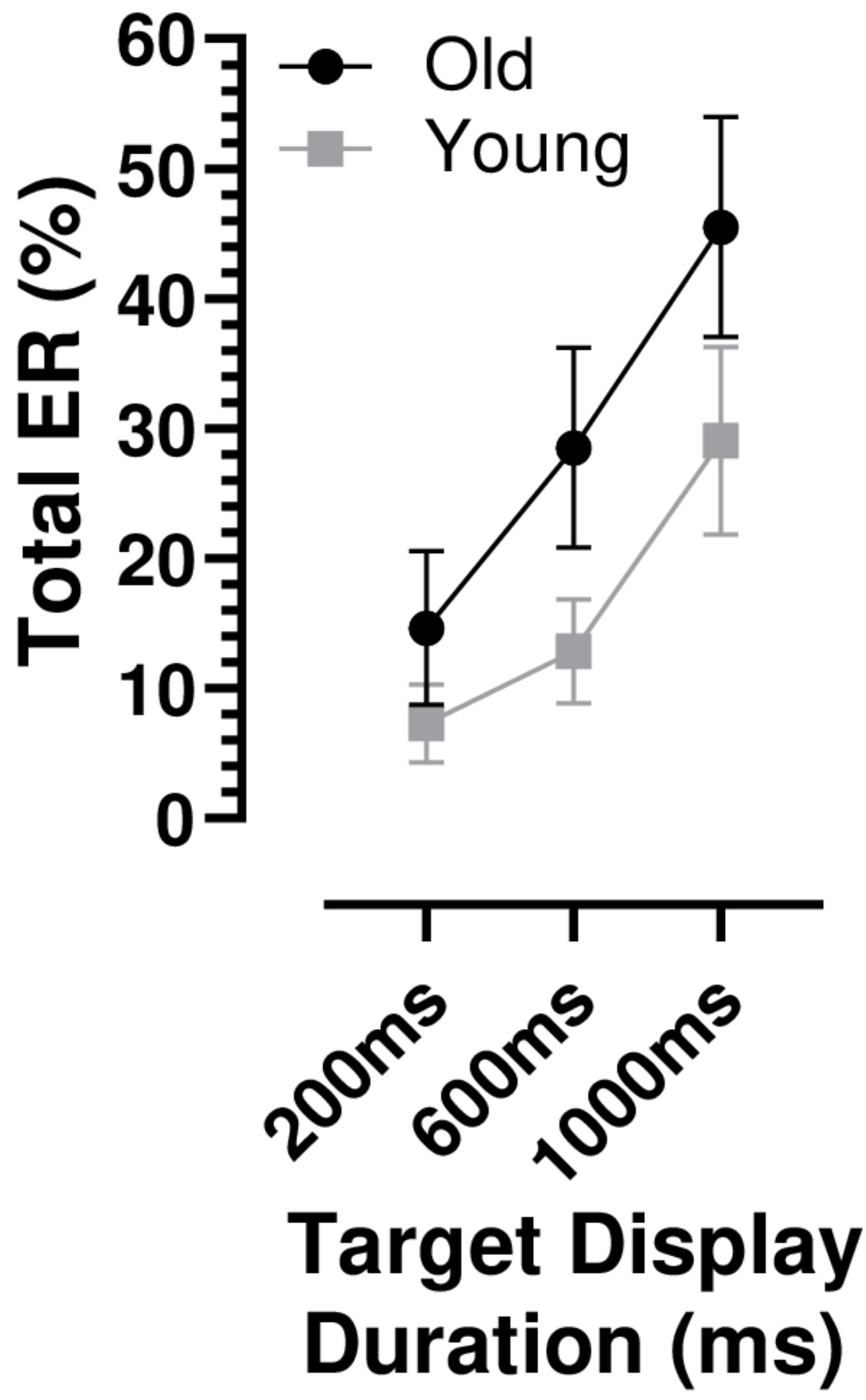
Figure 4

Average distribution plots for young $(A, B, C)$ and old $(D, E, F)$ participant groups.

Bin width $=50 \mathrm{~ms}$. For each participant, the $\%$ frequency distribution for all responses was calculated. The mean $( \pm 95 \% \mathrm{Cl})$ was then calculated for each bin across participants in each group. The black central line is plotted through the each mean bin value, while the grey range shows $\pm 95 \% \mathrm{Cl}$. The data were not smoothed. Target onset is at -200 for $200 \mathrm{~ms}$ TDD $(A, D),-600$ for $600 \mathrm{~ms}$ TDD $(B, E)$ and $-1000 m s$ for $1000 m s$ TDD $(C, F)$; target offset (the go signal) is at 0ms (vertical dotted line). Arrows mark peak bins in each distribution for both errors and correct responses, and the timing of that bin is also shown.

A. Y200ms

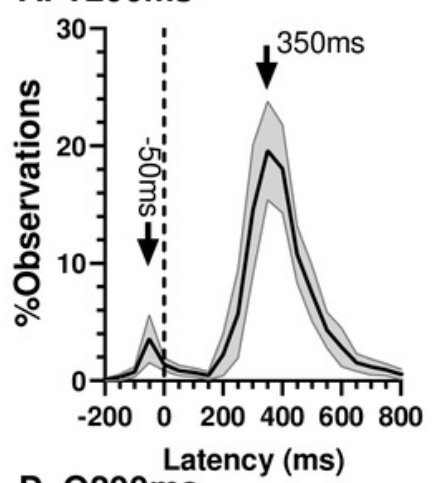

D. $0200 \mathrm{~ms}$

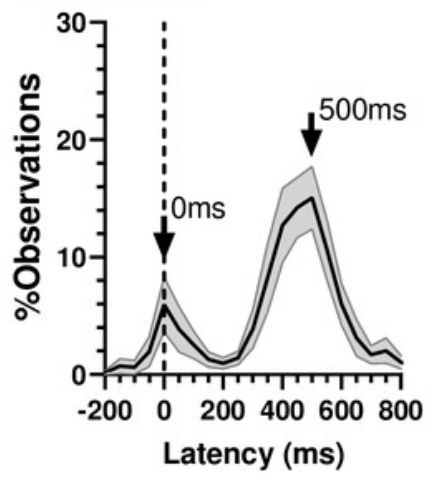

B. Y600ms

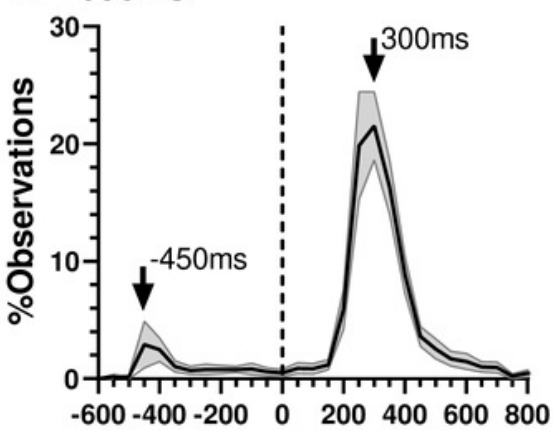

E. $0600 \mathrm{~ms}$

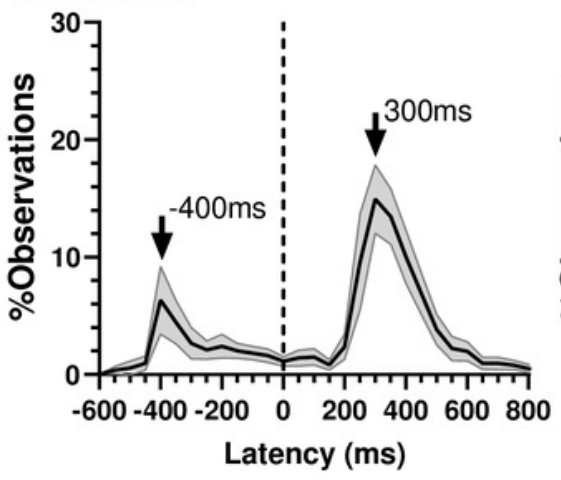

C. $\mathrm{Y} 1000 \mathrm{~ms}$

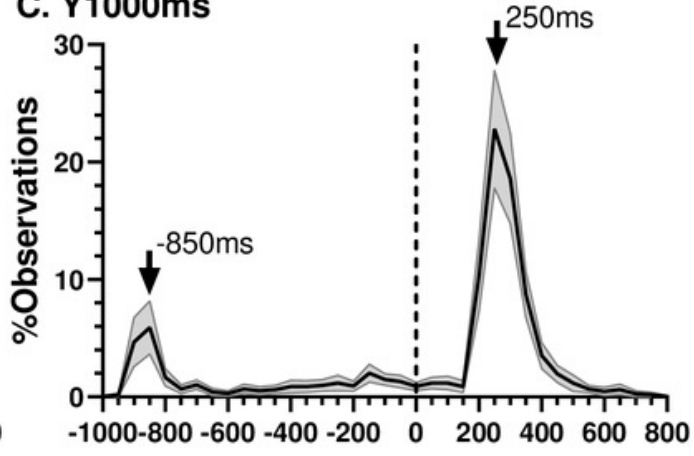

F. $01000 \mathrm{~ms}$

Latency (ms)

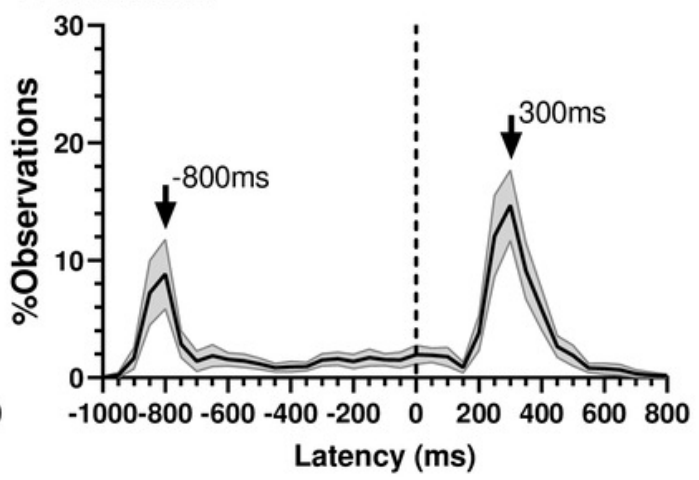


Figure 5

Peak and residual error rates

Mean $( \pm 95 \% \mathrm{Cl})$ MDOR peak error $(\mathrm{A})$ and residual error rates $(\mathrm{B})$ in old and young participant groups. The derivation of these error rates is described in the text.

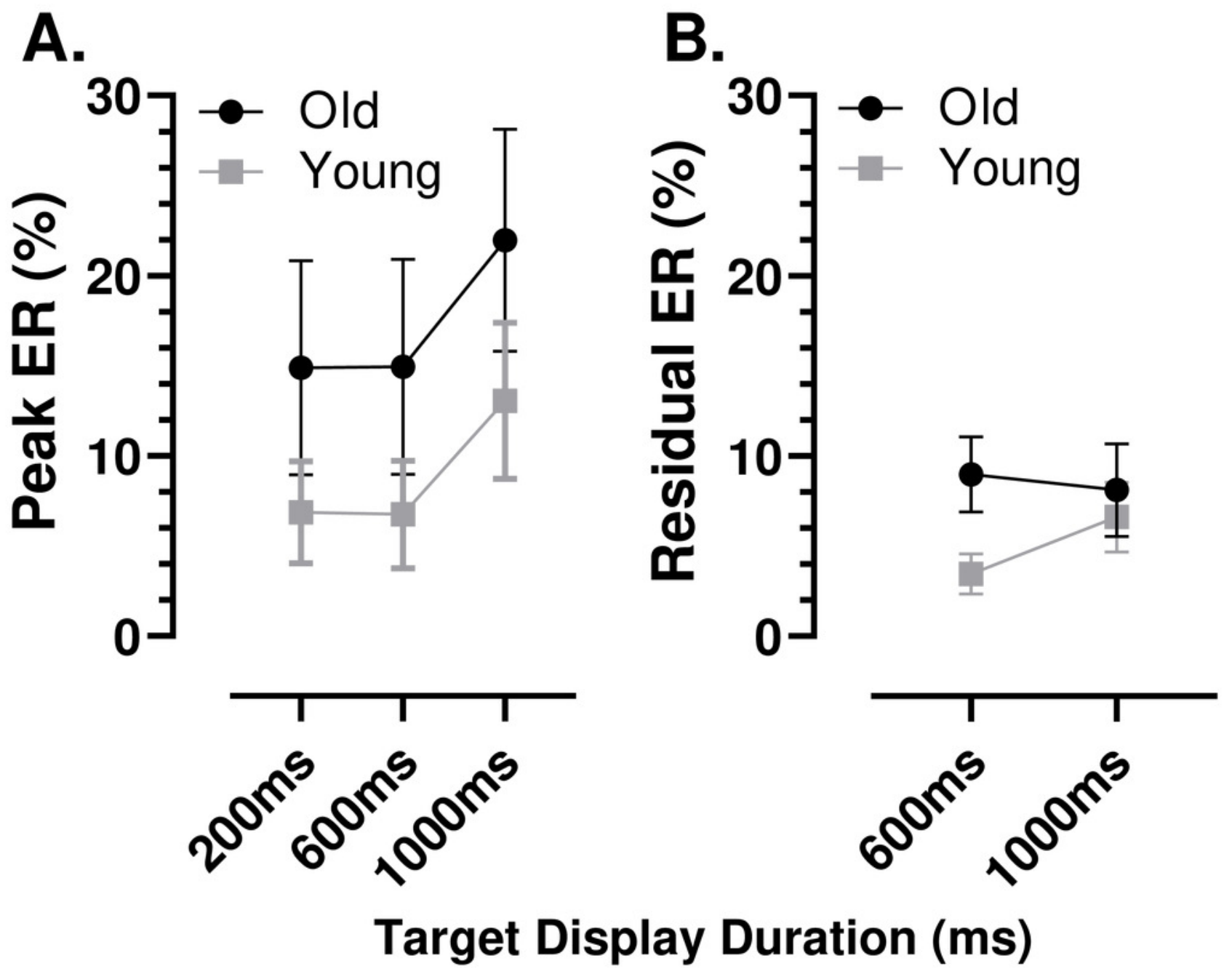


Figure 6

Latency of "peak" errors.

Mean $( \pm 95 \% \mathrm{Cl}$ ) latency of peak errors for old and young participant groups. Details of how these were calculated are in the text. 


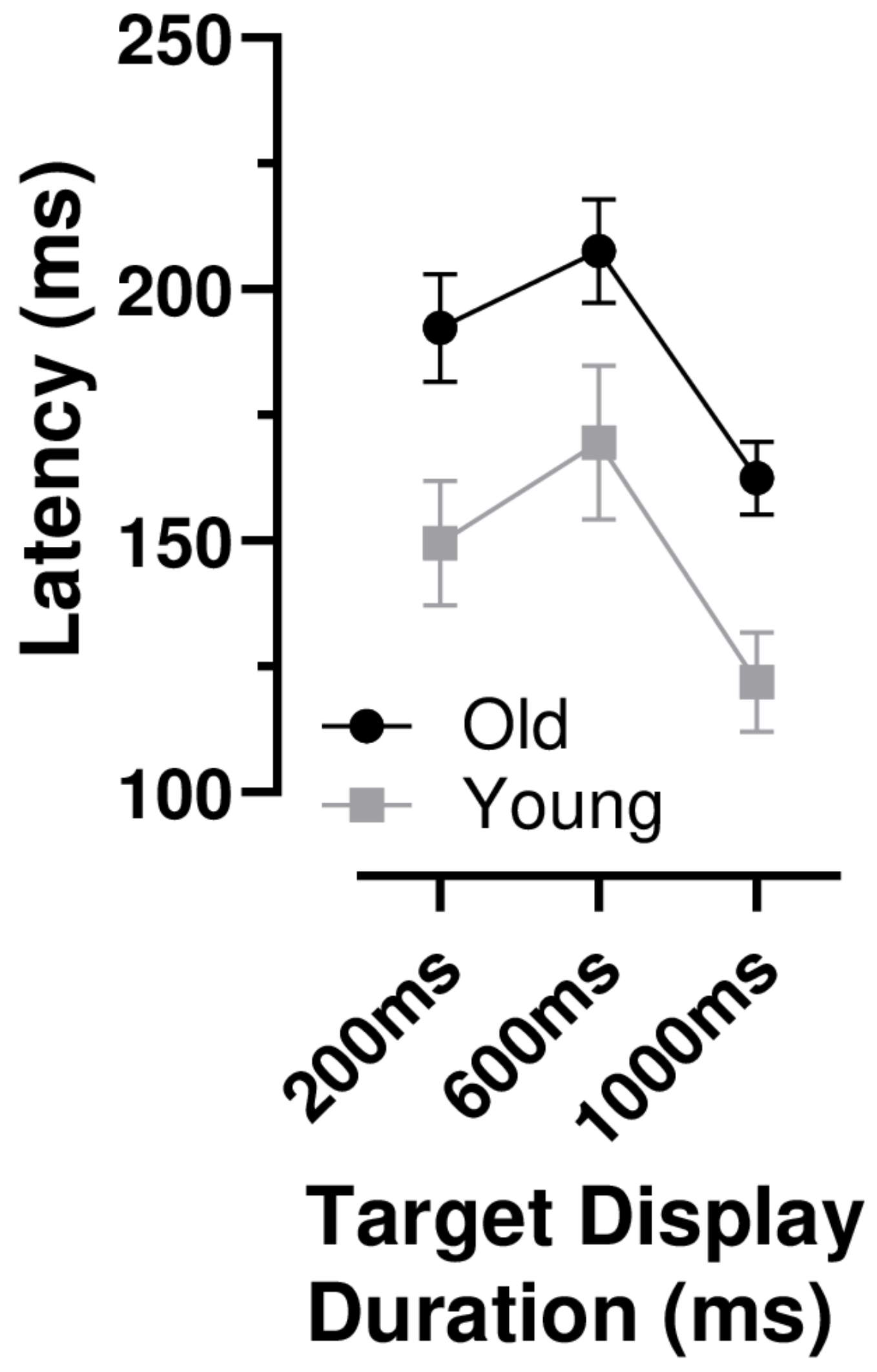




\section{Figure 7}

Correlation between MDOR peak error and prosaccade latencies.

Correlation between MDOR error saccade latency for saccades occurring in the peak error range and median pro-saccade latency calculated from the calibration task. (A) TDD 200ms; (B) TDD 600ms; (C) TDD 1000ms. On each plot the Pearson correlation coefficient is shown (with accompanying $\mathrm{p}$ value), calculated for the whole dataset (ie old and young data combined), although on each plot the groups can be distinguished (old: black circles; young: grey squares). Dashed line shows the line of equality $(x=y)$; points falling below this line indicate that the pro-saccade latency > MDOR error latency.
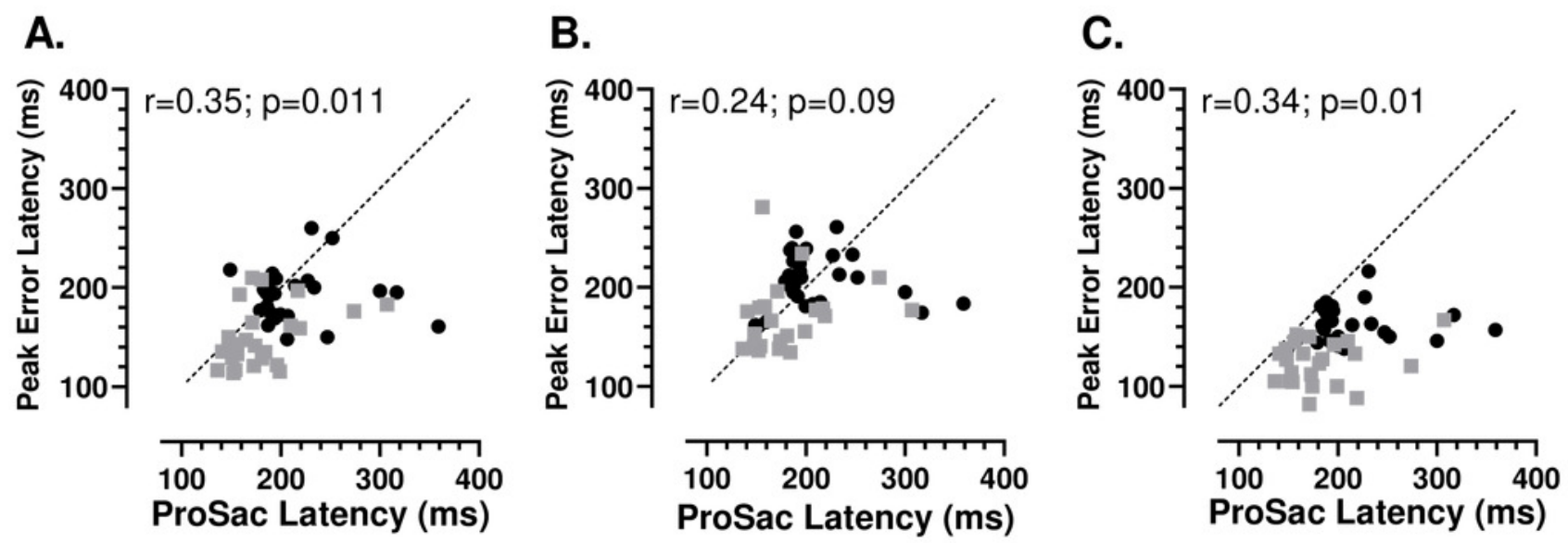
Figure 8

Relationship between error rates and latency of correct responses.

Plots of Error Rate (Total ER, ER $: A, C ;$ Peak ER, ER $\mathrm{pk}: \mathrm{B}, \mathrm{D})$ against latency for each participant in both young $(A, B)$ and old $(C, D)$ groups. In each plot, data from the three TDDs are distinguished: $200 \mathrm{~ms}$ gray symbols ; $600 \mathrm{~ms}$ unfilled symbols; $1000 \mathrm{~ms}$ black symbols. 

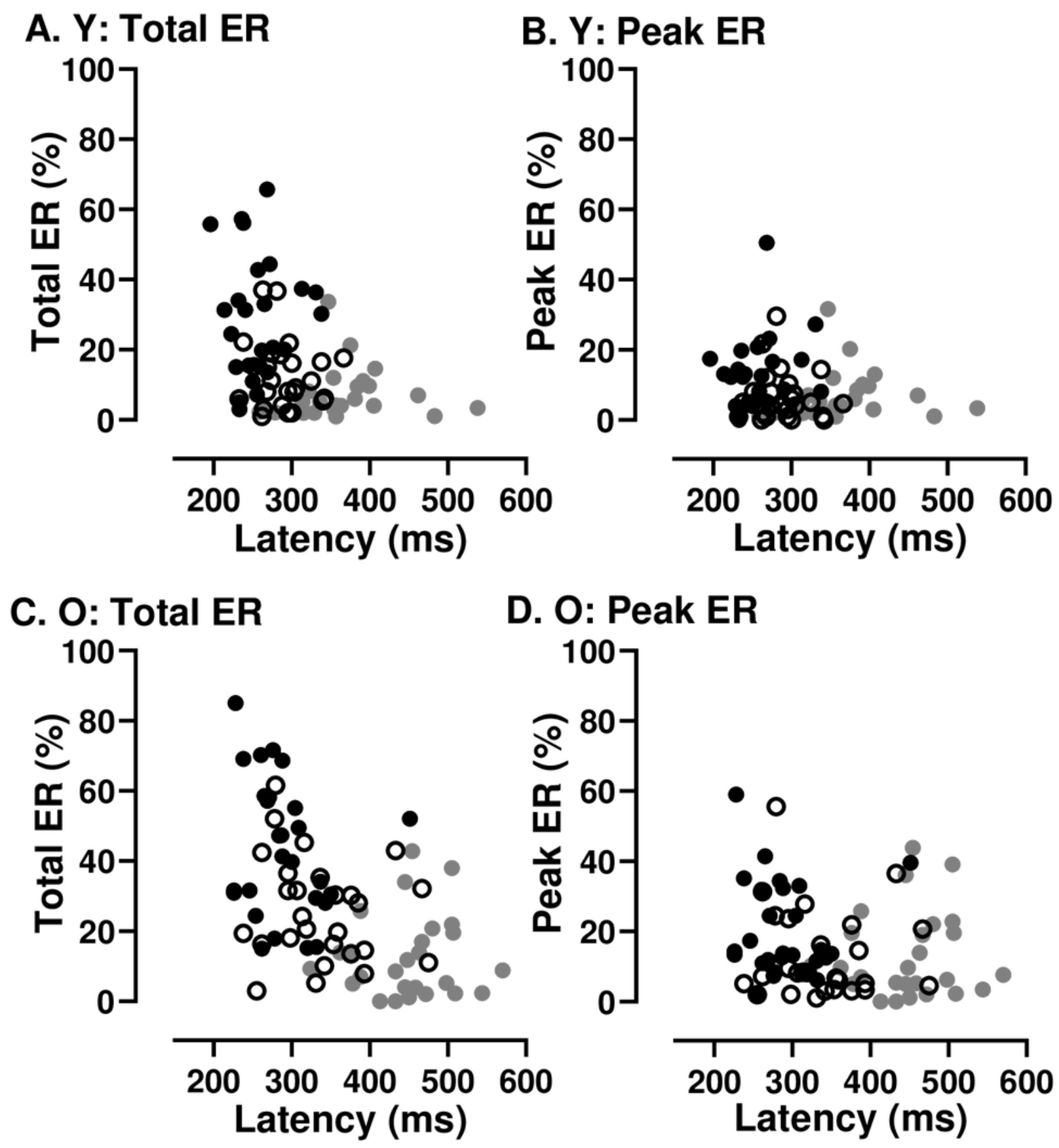

D. O: Peak ER
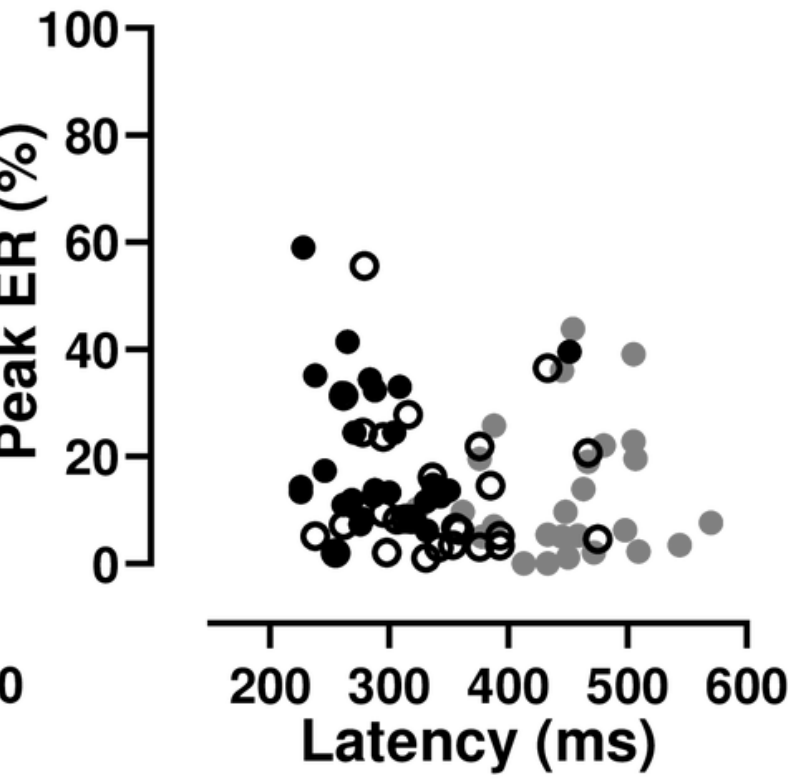
Figure 9

Comparison in MDOR performance between naïve and experienced participants.

Comparison of performance in the modified MDOR task (MDOR-M) between those old participants who were naïve to testing $(\mathrm{N})$ and those who had participated in a previous MDOR experiment with the original MDOR task (MDOR-O), and who had returned for testing (R). (A) Mean $( \pm 95 \% \mathrm{Cl})$ raw latency for correct responses. (B) Total error rate.

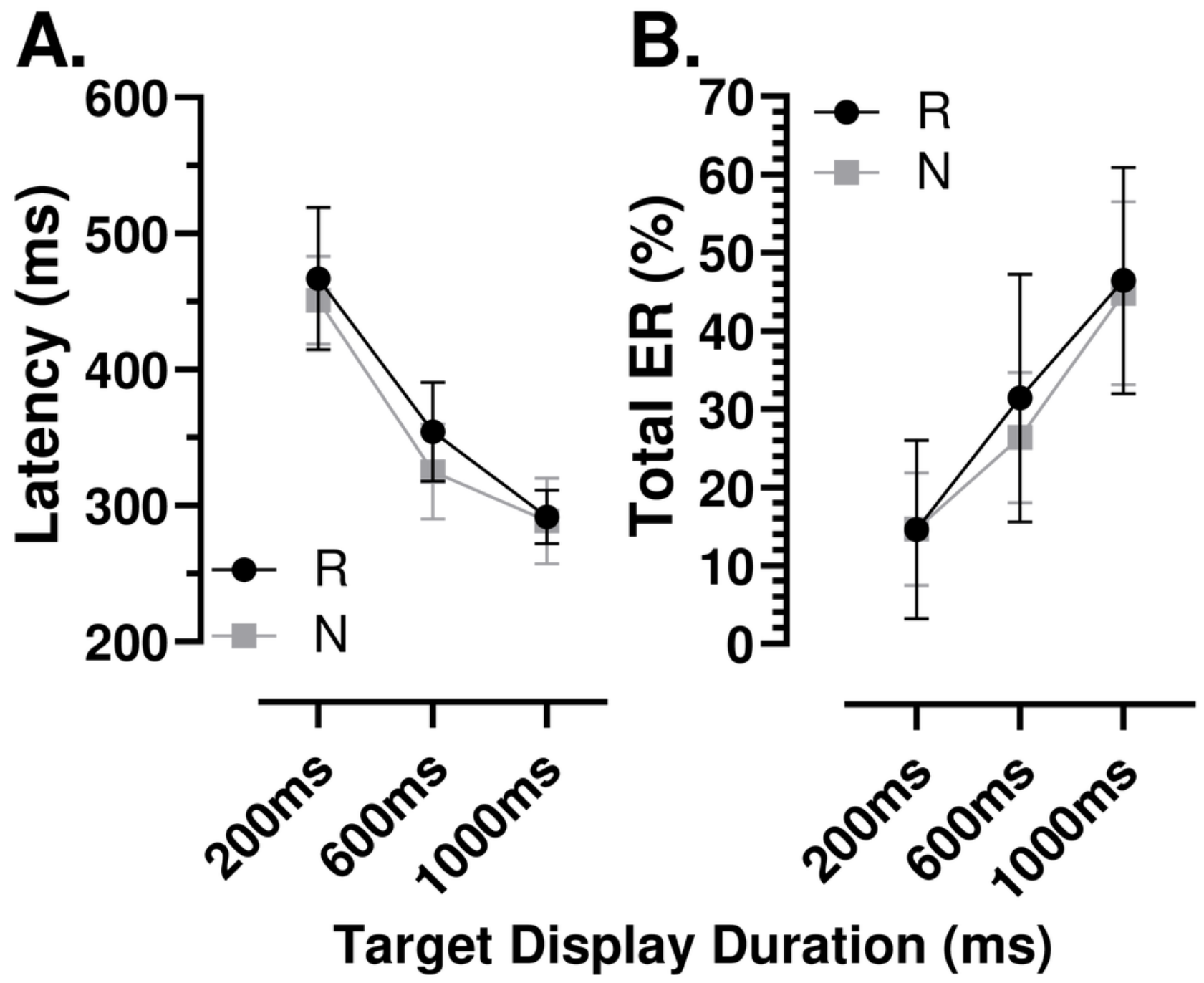




\section{Figure 10}

Comparison in perfomance between the original and modified MDOR tasks.

Comparison of performance in the original MDOR task (MDOR-O) and the modified task described in the current study (MDOR-M), Mean $( \pm 95 \% \mathrm{Cl})$ latency $(\mathrm{A}-\mathrm{C})$ and total error rate $(D-E)$ is shown for all participants for whom data were available $(A$ and $D ; n=24)$ and then divided into young ( $B$ and $E ; n=9)$ and old $(C$ and $F ; n=15)$ participants. 


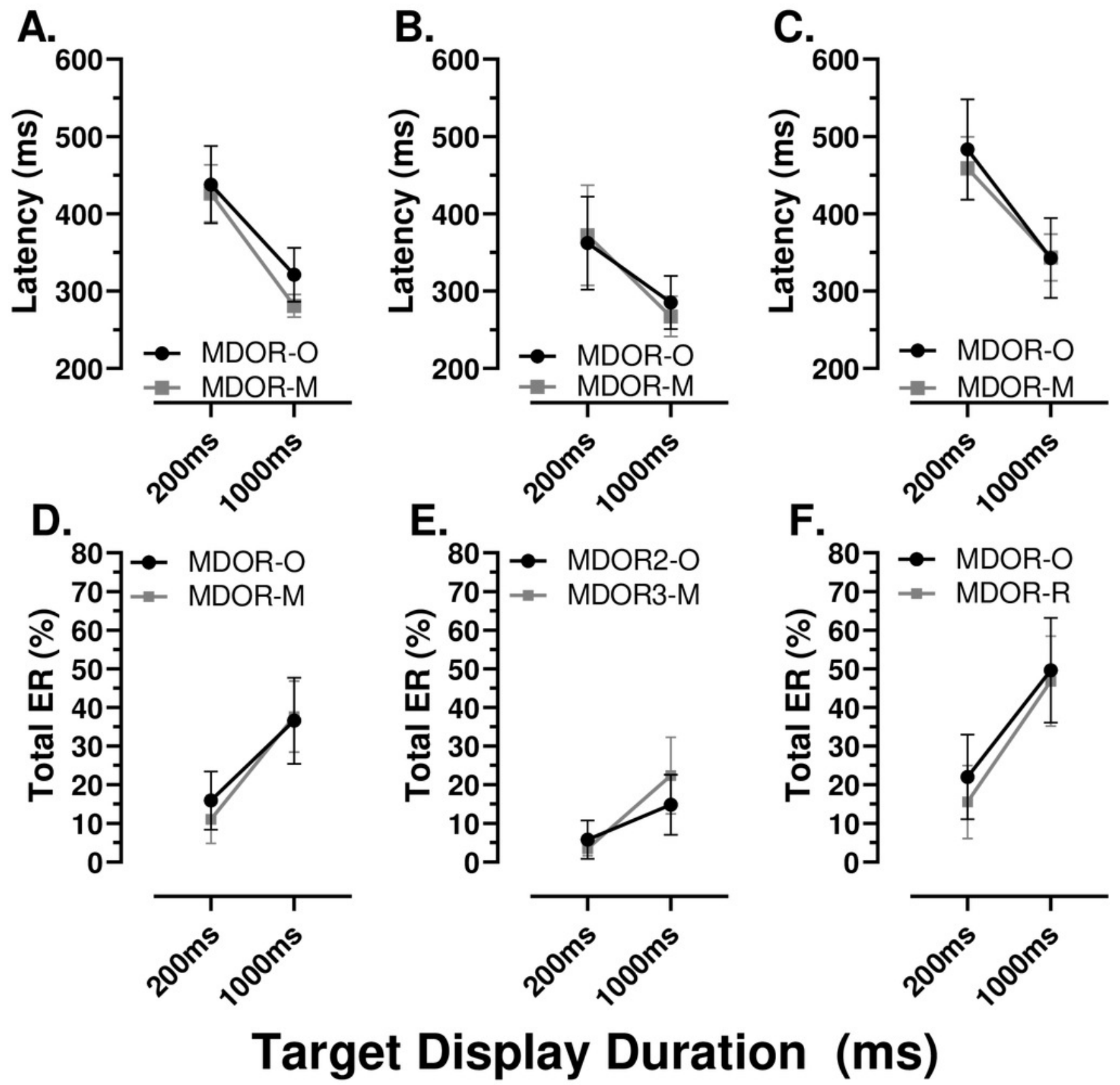

